\title{
Theory of induced magnetic moments and x-ray magnetic circular dichroism in Co-Pt multilayers
}

\author{
Claude Ederer, Matej Komelj, Manfred Fähnle,* and Gisela Schütz \\ Max-Planck Institut für Metallforschung, Heisenbergstrasse 1, D-70569 Stuttgart, Germany
}

(Received 8 May 2002; published 16 September 2002)

\begin{abstract}
For Co-Pt multilayers, the magnetic moments of the Pt atoms and the $\mathrm{x}$-ray magnetic circular dichroism (XMCD) spectra at the $L_{2}$ and $L_{3}$ edge of Pt are calculated by the ab initio density-functional electron theory. The calculated magnetization profile for an ideal Co-Pt interface is in part different from the profile obtained by x-ray resonant magnetic reflectometry for the real interface. Some of the assumptions that are commonly adopted to determine the magnetic moments from the XMCD spectra via the sum rules are critically assessed for the Co-Pt system. It is shown that the orbital sum rule is strongly violated near the Co-Pt interface whereas the spin sum rule is approximately fulfilled provided the magnetic dipole term $\left\langle T_{z}\right\rangle$ is included in the analysis.

DOI: 10.1103/PhysRevB.66.094413

PACS number(s): 75.70.Cn, 75.90.+w
\end{abstract}

\section{INTRODUCTION}

Multilayered magnetic films consisting of magnetic and nonmagnetic components have attracted considerable interest in the last years, especially because of the many potential technological applications in magnetic sensors, magnetoelectro and spin-electronic devices and for magneto-optical recording. Thereby the multilayer Co-Pt system is especially interesting because the $\mathrm{Pt}$ atoms that are paramagnetic in the bulk are significantly polarized close to the Co-Pt interface due to their large Stoner factor. As a result, the Pt atoms contribute to any magnetic property of the system, for instance, to the total magnetization or to the magneto-optical response. ${ }^{1}$ Because the polarization of the $\mathrm{Pt}$ atoms decreases with increasing distance from the interface, ${ }^{2}$ the properties of the multilayer system may be efficiently tuned by varying the layer thicknesses. For instance, one could try to achieve a spin injection from the Co layer into $\mathrm{Si}$ via a Pt buffer layer, which prevents the formation of Co silicide. Because the spin injection thereby is mediated by the induced spinpolarization of the Pt atoms, which depends on their distance from the Co-Pt interface, it is possible to vary the matching conditions for the spin injection by varying the thickness of the Pt buffer layer. From this point of view it becomes clear that a knowledge of the magnetization profile in the Pt layer is essential.

Unfortunately, it is very difficult to get a layer-resolved experimental information on the induced $\mathrm{Pt}$ magnetization profile. First, one has to use an element-specific method such as the $\mathrm{x}$-ray magnetic circular dichroism (XMCD) spectroscopy $^{3-7}$ or the $\mathrm{x}$-ray resonant magnetic scattering ${ }^{2}$ (XRMS) to distinguish between the contributions of Co and Pt. Second, there must be a procedure to get layer-resolved information from the total signal. Two methods have been used for a layer-dependent analysis of the XMCD results. In the first method ${ }^{5}$ one or more specific Pt layers are replaced by Ir layers, hoping that the induced magnetic moment of the Ir atom (which is the neighbor of the Pt atom in the periodic table) is essentially the same as the Pt atom would have in a corresponding Pt layer. In the second method ${ }^{6,7}$ the average Pt moments of multilayers with different Pt layer thicknesses are subtracted from each other. Assuming that the moments of the various Pt monolayers depend just on the distance from the Co-Pt interface but not on the Pt layer thickness, this yields the Pt magnetization profile. In both methods the orbital moments and the spin moments are obtained from the XMCD spectra by use of the so-called sum rules. ${ }^{8,9}$ For the XRMS study ${ }^{2}$ the Pt magnetization profile was deduced from the measured asymmetry ratio in the resonant reflectivity and from modeling with a magnetically modified Parratt formalism that divides the Pt layer into 0.1-nm-thick sheets and assigns a magnetic optical constant to each sheet. The free parameter in the modeling is the change in this constant of each sheet due to the induced magnetization in this sheet.

In the present paper we calculate by the ab initio densityfunctional electron theory the profiles of the induced orbital moments and spin moments in the Pt layers of various $\mathrm{Co}_{m}-\mathrm{Pt}_{n}$ multilayers, where $m$ and $n$ denote the numbers of Co monolayers and Pt monolayers in the Co and Pt layers, respectively. There are two objectives of the present study. First, the calculations are performed for ideal (i.e., atomically flat) Co-Pt interfaces. From a comparison of the theoretical results with the experimental data one may get an idea of how the real interfaces in the considered experiments looked like. Second, we want to consider some general critical issues concerning the analysis of XMCD spectra in multilayers. The analysis is always based on the sum rules which are not strictly valid but which rely on some approximations. ${ }^{10}$ By comparing the directly calculated moments with the moments obtained via the sum rules from the calculated XMCD spectra, we can check the validity of the sum rules at interfaces. Furthermore, the sum rule from which the spin moment is obtained ${ }^{9}$ contains a term involving the expectation value of the spin magnetic dipole operator $\hat{T}$. In transition metals, this term is negligibly small for cubic symmetry but nonzero otherwise, e.g., in the neighborhood of surfaces or interfaces. Because it is very difficult to obtain this term experimentally, it is usually neglected when calculating the spin moment from the spin sum rule. In the present paper, the importance of the $\hat{T}$ term will be investigated for layers close to the interface. For surfaces of $\mathrm{Fe}, \mathrm{Co}$, and $\mathrm{Ni}$, a corresponding check of the validity of the sum rules and the importance of the $\hat{T}$ term has been performed by $\mathrm{Wu}$ and Freeman. ${ }^{11}$ It has been found that the spin sum rule is less well satisfied than the orbital sum rule which 
results in errors up to $50 \%$ for the spin moment at the $\mathrm{Ni}$ (001) surface, and that it is essential to include the $\hat{T}$ term. Finally, we will check the assumption underlying the second method of the layer-resolved analysis of the XMCD spectra, i.e., that the moments of the various Pt monolayers depend just on the distance from the Co-Pt interface but not on the thickness of the whole Pt layer.

\section{DETAILS OF THE CALCULATION}

We consider multilayer $\mathrm{Co}_{m}-\mathrm{Pt}_{n}$ systems with $m=2$ and $n=3,7,9,13$ with atomically flat and unrelaxed (111) interfaces and perpendicular magnetization. Some test calculations for $m=3$ were also performed, yielding nearly the same results for the Pt atoms as for $m=2$. As lattice constant we took the experimental bulk value of fcc Pt $(3.92 \AA)$. The calculations within the framework of the density-functional theory and the local-spin-density approximation ${ }^{12}$ were performed by the tight-binding linear-muffin-tin-orbital method (LMTO) in the atomic sphere approximation ${ }^{13}$ (ASA) with our code which allows to consider noncollinear spin arrangements ${ }^{14,15}$ and in which we have implemented the spin-orbit coupling, as well as with the WIEN97 code $^{16}$ which adopts the full-potential linearized-augmented-planewave method $^{17}$ (FLAPW) and in which the spin-orbit coupling and the tools for the calculation of magneto-optical effects and XMCD spectra have been implemented. ${ }^{18,19}$ In most of the figures we represent data for $m=2, n=7$. Again, our test calculations have shown that the results for larger (but not smaller) $n$ are very similar. By the FLAPW method we have performed also some calculations where we have taken into account the effect of layer relaxations at the interface, but there was nearly no influence on the moments and on the spectra. The spin-orbit coupling was taken into account self-consistently. The absorption coefficients $\mu^{+}(\epsilon)$, $\mu^{-}(\epsilon)$, and $\mu^{0}(\epsilon)$ for right-circular, left-circular, and $z$-axis polarization as function of the photon energy $\epsilon$ were calculated by use of Fermi's golden rule in nonrelativistic dipole approximation which requires the evaluation of matrix elements for the operator $\hat{\mathbf{p}} \cdot \mathbf{e}$ with $\mathbf{e}=\mathbf{e}^{-}, \mathbf{e}^{+}$, or $\mathbf{e}^{0}$ denoting the polarization vector of the light. For the FLAPW method the principle procedure is described in Refs. 20,21.

As discussed below, the absorption signal is in most cases strongly dominated by transitions to one type of $n l$ states. For the Pt $L_{2}$ and $L_{3}$ spectra the dominant transition is to unoccupied $5 d$ states, whereas the additional contributions of $n s$ states with $n \geqslant 6$ and $n d$ states with $n>5$ (which are all allowed in dipole approximation) are weaker. There are further additional contributions that come into play when going beyond the dipole approximation, which also should be less important. The dominant contribution is mainly responsible for the so-called white line around the absorption edge, whereas the additional contributions result in a long tail beyond the white line which is often plateaulike. This is especially pronounced for the case of $\mathrm{Pt}$ where the plateau is nearly as high as the white line., ${ }^{4,5}$ The physical reason for this is that the unoccupied $n s, n d$ states of the free Pt atom have high energies that are close to each other, and they are spatially strongly extended. Therefore, bringing the Pt atoms

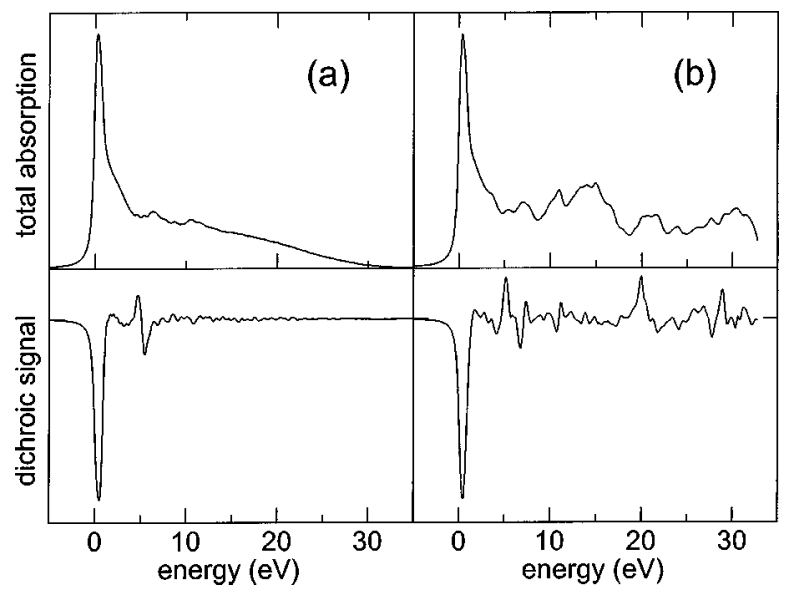

FIG. 1. The total absorption signal $\mu_{\mathrm{t}}(\epsilon)$ (top) and the dichroic signal $\mu_{\mathrm{c}}(\epsilon)$ (bottom) for the $L_{2}$ edge of the Pt interface layer in $\mathrm{Co}_{2}-\mathrm{Pt}_{7}$ system as calculated by the LMTO method (a) and the FLAPW method (b). For better visualization the data was artificially broadened with a Lorentzian with a width of $0.3 \mathrm{eV}$.

together to a crystal leads to a strong overlap of the various resulting bands and thus to a high density of unoccupied states for the absorption signal beyond the white line. In contrast, the difference signal $\mu_{\mathrm{c}}(\epsilon)=\mu^{+}(\epsilon)-\mu^{-}(\epsilon)$ usually is well localized around the white line (Fig. 1). The physical reason for this localization is that the states involved in the nondominant transitions usually are strongly itinerant so that at a given energy the difference between the density of states for spin up and spin down is small. Because $\mu_{\mathrm{c}}(\epsilon)$ is basically determined by this difference ${ }^{4,5,10}$ there is only a small contribution of strongly itinerant states to $\mu_{\mathrm{c}}(\epsilon)$.

The LMTO and the FLAPW code use a set of energyindependent basis functions that are designed to describe the wave functions for a certain energy range as accurately as possible. This energy range is around a linearization energy that we choose according to the common practice as being the center of gravity of the occupied $5 d-6 s-6 p$ band. The so-obtained basis set should be able to describe rather accurately also the states slightly above the Fermi level, but it becomes more and more less complete when going to higher energies. In contrast, in a calculation based on a nonlinearized Korringa-Kohn-Rostocker (KKR) method the functions with angular momentum $l$ properly account for the $n l$ states at all energies. As a result, KKR calculations for the $L_{2}$ and $L_{3}$ edge of Pt yield ${ }^{22,23}$ a plateaulike extension of the absorption signal similar to the experiment, whereas in the LMTO and FLAPW calculation the signal decreases strongly above the white line (Fig. 1). Obviously, the latter two calculations miss many of the high-lying strongly itinerant states. Altogether, the usual LMTO and FLAPW codes therefore give reliable results for $\mu_{\mathrm{c}}(\epsilon)$ but they do not necessarily describe correctly the absorption signals $\mu^{+}, \mu^{-}$, and $\mu^{0}$. For the analysis of the spectra by use of the sum rules (see below) the contributions of the high-lying strongly itinerant states have to be removed anyway, and therefore for this objective it is not a problem that the two codes miss many of these states in Pt.

In our calculations the $d$ band extends to high energies 


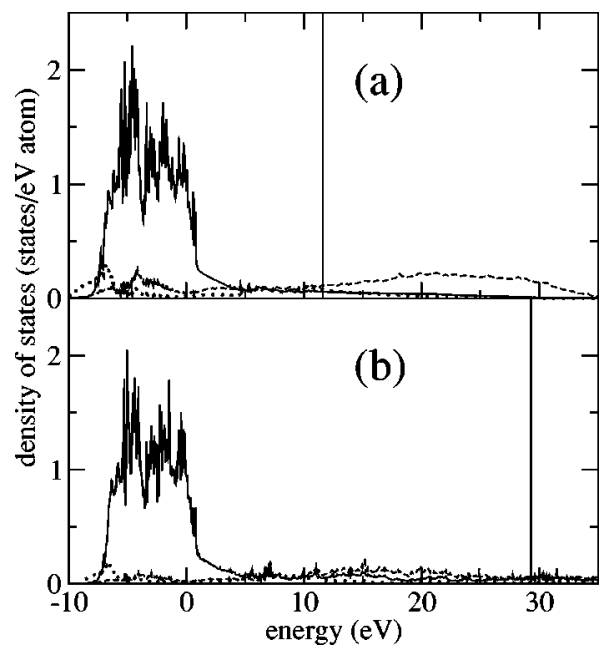

FIG. 2. The orbital-resolved density of states for the Pt interface layer in $\mathrm{Co}_{2}-\mathrm{Pt}_{7}$ system as calculated by the LMTO method (a) and the FLAPW method (b). The Fermi level is $E_{\mathrm{F}}=0$. The vertical line gives the upper edge of the " $5 d$ band," see text. Solid lines: $d$ states. Broken lines: $p$ states. Dotted lines: $s$ states.

(Fig. 2) because of the contributions of $n d$ states with $n$ $>5$. We have then defined the upper edge of the " $5 d$ band" in such a way that this band contains 10 electrons per atom (Fig. 2).

The induced spin moments $m_{s}=-\mu_{\mathrm{B}}\left\langle\sigma_{z}\right\rangle$ and orbital moments $m_{l}=-\mu_{\mathrm{B}}\left\langle l_{z}\right\rangle$ for the various $\mathrm{Pt}$ monolayers were calculated directly as well as via the orbital sum rule ${ }^{8}$ and the spin sum rule from the absorption spectra at the $\mathrm{Pt} L_{2}$ and $\mathrm{Pt}$ $L_{3}$ edge,

$$
\begin{gathered}
\left\langle l_{z}\right\rangle=\frac{2 I_{\mathrm{m}} N_{\mathrm{h}}}{I_{\mathrm{t}}}, \\
\left\langle\sigma_{z}\right\rangle=\frac{3 I_{\mathrm{s}} N_{\mathrm{h}}}{I_{\mathrm{t}}}-7\left\langle T_{z}\right\rangle, \\
I_{\mathrm{m}}=\int_{E_{\mathrm{F}}}^{E_{\mathrm{c}}}\left[\left(\mu_{\mathrm{c}}\right)_{L_{3}}+\left(\mu_{\mathrm{c}}\right)_{L_{2}}\right] d \epsilon, \\
I_{\mathrm{s}}=\int_{E_{\mathrm{F}}}^{E_{\mathrm{c}}}\left[\left(\mu_{\mathrm{c}}\right)_{L_{3}}-2\left(\mu_{\mathrm{c}}\right)_{L_{2}}\right] d \epsilon, \\
I_{\mathrm{t}}=\int_{E_{\mathrm{F}}}^{E_{\mathrm{c}}}\left[\left(\mu_{\mathrm{t}}\right)_{L_{3}}+\left(\mu_{\mathrm{t}}\right)_{L_{2}}\right] d \epsilon
\end{gathered}
$$

with $\mu_{\mathrm{c}}=\mu^{+}-\mu^{-}$and $\mu_{\mathrm{t}}=\mu^{+}+\mu^{-}+\mu^{0}$. Here $N_{\mathrm{h}}$ is the number of $d$ holes and $\left\langle T_{z}\right\rangle$ is the expectation value of the magnetic dipole operator

$$
\hat{T}_{z}=\frac{1}{2}[\boldsymbol{\sigma}-3 \hat{\mathbf{r}}(\hat{\mathbf{r}} \cdot \boldsymbol{\sigma})]_{z},
$$

where $\boldsymbol{\sigma}$ denotes the vector of the Pauli matrices. The quantities $E_{\mathrm{F}}$ and $E_{\mathrm{c}}$ denote the Fermi energy and a cutoff energy (see below). In the LMTO-ASA method the term $\left\langle T_{z}\right\rangle$ is determined from the nonspherical charge and spin density obtained after the last iteration step. Because this charge and spin density is calculated from an ASA potential that is spherically averaged in each atomic sphere before starting an iteration step, the influence of the nonspherical parts of the effective potential within the spheres on the asphericity of the charge and spin density is neglected. In contrast, in the FLAPW calculation the full asphericity of the effective potential is taken into account, and therefore the results for $\left\langle T_{z}\right\rangle$ from the two calculational methods will be slightly different.

The derivations ${ }^{8,9,24}$ of the sum rules, Eqs. (1) and (2), are based on the dipole approximation and on several other approximations listed in Sec. 2.7 of Ref. 10. One major approximation is that the above discussed additional contributions to the absorption signal can be neglected. As already outlined, these additional contributions will have only a weak influence on the dichroic signal but they would critically enter the integrations in the denominators of Eqs. (1) and (2). For a consistent test of the sum rules by theory one therefore should take into account ${ }^{11}$ for all the quantities appearing in Eqs. (1) and (2) only the dominant $n l$ contributions. We will report the results of such a calculation in the following section.

\section{TEST OF THE SUM-RULE ANALYSIS OF EXPERIMENTAL DATA}

In the present section we want to test for the multilayer Co-Pt system some assumptions and approximations involved in an experimental determination of the induced $\mathrm{Pt}$ moments via the sum rules. All calculations are performed for the multilayer $\mathrm{Co}_{2}-\mathrm{Pt}_{7}$ system. Test calculations have shown that the results are nearly identical to those obtained for $\mathrm{Co}_{2}-\mathrm{Pt}_{13}$ system.

\section{A. Restriction to the dominant $5 d$ contributions}

The experimental absorption spectra do not contain just the dominant $5 d$ contributions but all the additional contributions discussed in Sec. II, and these contribute significantly to the integrations in the denominators of Eqs. (1) and (2). Therefore it is attempted to remove the additional contributions ("background subtraction") at least approximately. The hope is that the " $5 d$-magnetic moments" obtained from these corrected spectra via the sum rules are good estimates for the "real moments" including all contributions. This hope implies two assumptions. The first assumption is that the non- $5 d$ contributions to the magnetic moments are indeed very small. Figures 3 and 4 exhibit the magnetic moments calculated directly from the bandstructure calculations. It becomes clear that the $5 d$ orbital moments (small filled circles) are indeed nearly indistinguishable from the real orbital moments (big open circles), whereas the $5 d$ spin moments are consistently larger than the real spin moments, the difference being about $9 \%$ of the total moment for the Pt interface layer in the LMTO calculation and $4 \%$ in the FLAPW calculation. The second hope is that the additional approximations involved in the derivation of the sum rules (see Sec. II) are indeed well fulfilled. To test for this, we calculate the absorption signals and the dichroic 


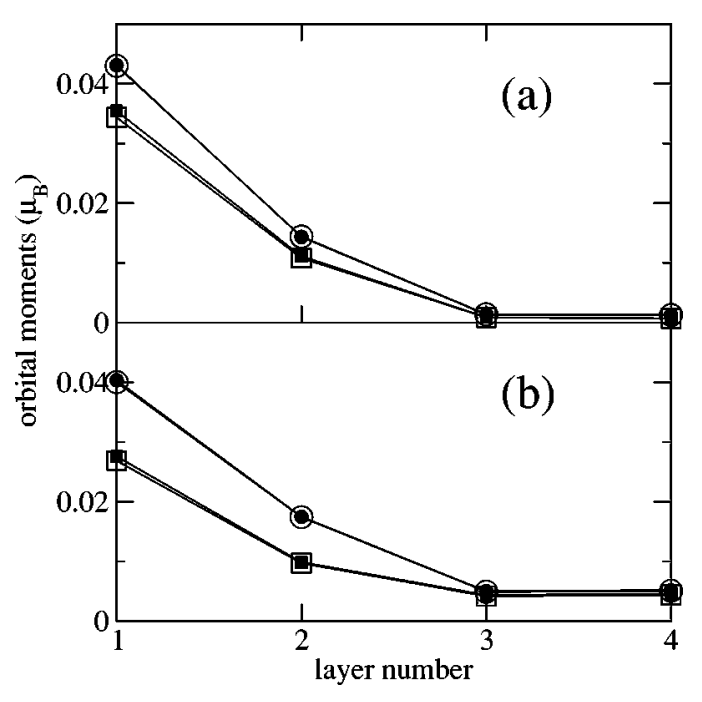

FIG. 3. Test of the orbital sum rule for the various Pt monolayers $l$ in $\mathrm{Co}_{2}-\mathrm{Pt}_{7}$ system ( $l=1$ denotes the interface layer) by LMTO (a) and FLAPW (b) calculations. Big open circles: directly calculated real moments (see text). Small filled circles: directly calculated $5 d$ moments (see text). Big open squares: estimates of the real moments by the orbital sum rule (see text), cutoff energy $E_{\mathrm{c}}$ is the upper edge of the $d$ band. Small filled squares: estimates of the $5 d$ moments by the orbital sum rule (see text).

signal as well as the $\left\langle T_{z}\right\rangle$ term by taking into account only the $5 d$ contributions. With these corrected theoretical spectra we then obtain via the sum rules estimates for the $5 d$ orbital and $5 d$ spin moments (small filled squares), which we com-

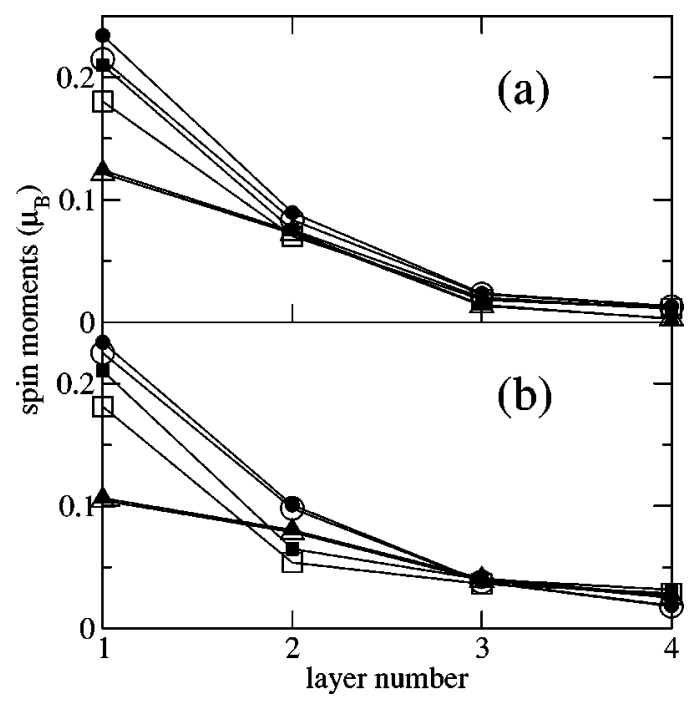

FIG. 4. Test of the spin sum rule for the various Pt monolayers $l$ in $\mathrm{Co}_{2}-\mathrm{Pt}_{7}$ system ( $l=1$ denotes the interface layer) by LMTO (a) and FLAPW (b) calculations. Big open circles: directly calculated real moments. Small filled circles: directly calculated $5 d$ moments. Big open squares: estimates for the real moments by the spin sum rule, including the $\left\langle T_{z}\right\rangle$ term. Small filled squares: estimates of the $5 d$ moments by the spin sum rule, including the $\left\langle T_{z}\right\rangle$ term. Big open triangles: estimates for the real moments by the spin sum rule, excluding the $\left\langle T_{z}\right\rangle$ term. Small filled triangles: estimates of the $5 d$ moments by the spin sum rule, excluding the $\left\langle T_{z}\right\rangle$ term. pare in Figs. 3 and 4 with the directly calculated $5 d$ moments. A good agreement between the two sets of data would indicate that the additonal approximations involved in the derivation of the sum rules are well fulfilled. It becomes clear from these figures that this holds at least approximately for the spin sum rule but definitely not for the orbital sum rule. Therefore, even if no other problems were involved in the experimental data analysis one should caution when trying to determine induced orbital moments at interfaces via the orbital sum rule.

Whereas in theory the background subtraction is straightforward, this is not the case for the experiments. The following approaches are usually adopted.

(1) A step function (or an arctan curve) is subtracted which is fitted to the plateau regime. For Pt this type of analysis may be critical ${ }^{25}$ because due to the high plateaulike regime the error in the determination of the steplike background may be quite large.

(2) The $L_{2}$ and $L_{3}$ edges of Pt are compared with those of $\mathrm{Au}$ measured under similar experimental conditions. ${ }^{25}$ The idea is that $\mathrm{Au}$ is electronically similar to $\mathrm{Pt}$ with the main exception that the $5 d$ band of Au is full in a first approximation whereas it is not completely full for Pt, so that an appropriate subtraction of the Au spectrum will isolate the $5 d$ part of the Pt spectrum. Our critcism of this procedure is that even in $\mathrm{Au}$ there are indeed holes in the $5 d$ band (see, e.g., Ref. 26), and our test calculations have shown that the $5 d$ contribution to the absorption spectrum of $\mathrm{Au}$ is even larger than the $6 s$ contribution. The subtraction of the Au spectrum may be able to eliminate most of the contributions of the strongly itinerant $n d(n>5)$ and $n s(n \geqslant 6)$ states, but what is left is not necessarily a very good representation of the $5 \mathrm{~d}$ contribution in Pt.

(3) The contributions of the energetically high-lying strongly itinerant states to the denominators of Eqs. (1) and (2) are excluded by integrating only up to an energy cutoff $E_{\mathrm{c}}$. The value of $E_{\mathrm{c}}$ is not clear ad hoc. It has been suggested $^{11}$ to identify it with the energy where $\mu_{\mathrm{c}}(\epsilon)$ becomes acceptably close to zero. This procedure does not remove the contributions of the additional transitions in the energy range of the dominant transitions. The hope is that these contributions are small. To test for the influence of the $s$ states in the energy range of the " $5 d$ band" we have calculated (Figs. 3 and 4) the orbital and spin moments from the sum rules (thereby neglecting the $\left\langle T_{z}\right\rangle$ term for the spin sum rule as in most experiments), on the one hand, by taking into account exclusively the $5 d$ contributions to the spectra (small filled squares for the orbital moment and small filled triangles for the spin moment), and on the other hand, by including the $s$ contributions but using an energy cutoff $E_{\mathrm{c}}$ $=E_{\mathrm{d}}$ for the integrations in Eqs. (1) and (2) where $E_{\mathrm{d}}$ is the upper edge of the " $5 d$ band" (big open squares and big open triangles for the orbital and spin moments). Obviously there is only a weak influence of the $s$ contributions to the spectra in this energy range.

\section{B. The influence of the $\left\langle T_{z}\right\rangle$ term}

Because it is very hard to access the $\left\langle T_{z}\right\rangle$ term experimentally, ${ }^{27,28}$ it is often neglected for the determination 


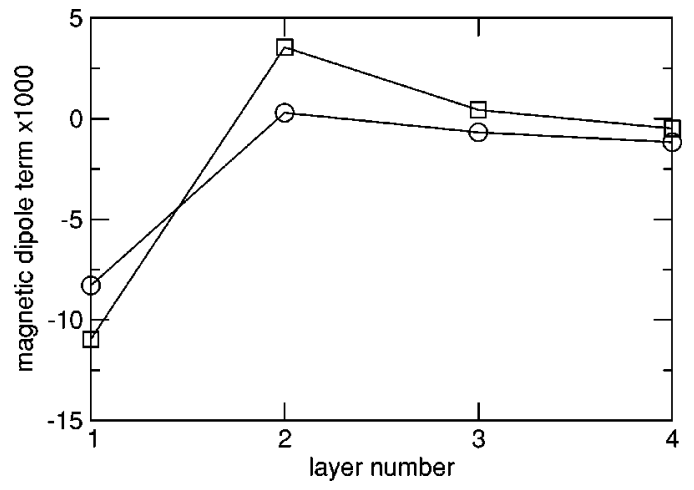

FIG. 5. Values for $\left\langle T_{z}\right\rangle$ for the various Pt layers in $\mathrm{Co}_{2}-\mathrm{Pt}_{7}$ system as obtained by the LMTO method (circles) and the FLAPW method (squares).

of the spin moment via the spin sum rule, even at interfaces. ${ }^{7}$ This term is negligible for cubic surroundings but it should become more and more important when approaching the interface. In Fig. 4 we represent the spin moments as obtained from the spin sum rule when including and when omitting the $\left\langle T_{z}\right\rangle$ term. The values obtained by omitting this term differ at the interface by about $30-50 \%$ from the values obtained when it is included. The spin moment obtained from the sum rule excluding the $\left\langle T_{z}\right\rangle$ term (big open triangles) is at the interface smaller than the directly calculated moment (big open circles) by about $40-50 \%$. This clearly demonstrates that for the interface the $\left\langle T_{z}\right\rangle$ term cannot be neglected in the spin sum rule.

In Fig. 5 we compare the $\left\langle T_{z}\right\rangle$ terms for the various $\mathrm{Pt}$ layers as obtained by the LMTO and the FLAPW calculation. The qualitative behavior is similar for both calculations but there are some quantitative discrepancies due to the different treatment of the on-site asphericity of the spin density (see Sec. II).

\section{Fixing the number of holes}

For the analysis of the experimental data via the sum rules the number $N_{\mathrm{h}}$ of holes in the $d$ band has to be fixed somehow. One way is to insert for $N_{\mathrm{h}}$ the value obtained by a band-structure calculation for a reference configuration that is similar to the real configuration under consideration. For instance, for an analysis of the Co-Pt multilayer one could insert the number of $5 d$ holes as calculated for fcc Pt. Indeed, we obtain by the LMTO calculation the values $N_{\mathrm{h}}=1.62 /$ atom for fcc $\mathrm{Pt}$ and $N_{\mathrm{h}}=1.68 /$ atom, 1.60/atom, 1.62/atom, and 1.62/atom for the first four Pt layers at the interface of the $\mathrm{Co}_{2}-\mathrm{Pt}_{7}$ multilayer, i.e., fixing the number $N_{\mathrm{h}}$ does not seem to be a critical problem.

\section{The ratios $m_{l} / m_{s}$}

From Eqs. (1) and (2) we obtain

$$
\frac{\left\langle l_{z}\right\rangle}{\left\langle\sigma_{z}\right\rangle}=\frac{m_{l}}{m_{s}}=\left[\frac{3}{2} \frac{I_{\mathrm{s}}}{I_{\mathrm{m}}}-\frac{7}{2} \frac{\left\langle T_{z}\right\rangle I_{\mathrm{t}}}{I_{\mathrm{m}} N_{\mathrm{h}}}\right]^{-1} .
$$

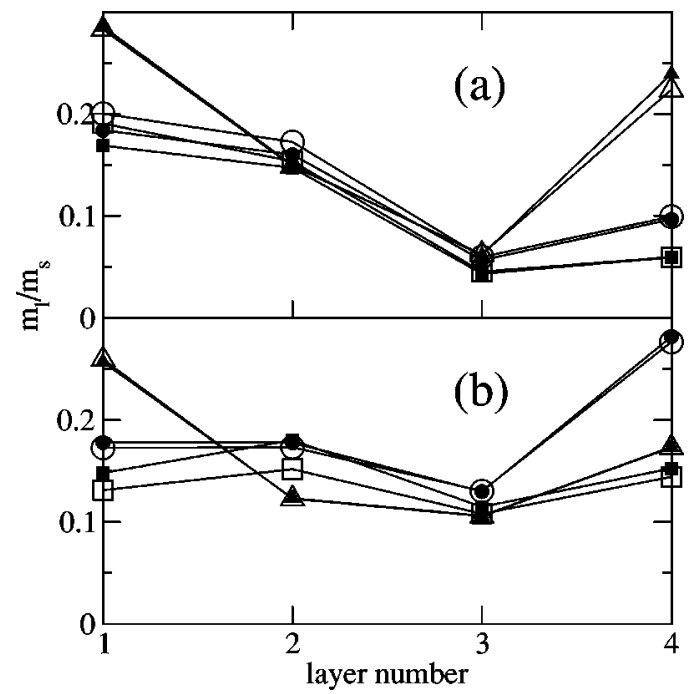

FIG. 6. Values for $m_{l} / m_{s}$ for the various Pt layers in $\mathrm{Co}_{2}-\mathrm{Pt}_{7}$ system as obtained by the LMTO method (a) and the FLAPW method (b). Big open circles: directly calculated real moments. Small filled circles: directly calculated $5 d$ moments. Big open squares: real moments via Eq. (7), including the $\left\langle T_{z}\right\rangle$ term. Small filled squares: $5 d$ moments via Eq. (7), including the $\left\langle T_{z}\right\rangle$ term. Big open triangles: real moments via Eq. (7), excluding the $\left\langle T_{z}\right\rangle$ term. Small filled triangles: $5 d$ moments via Eq. (7), excluding the $\left\langle T_{z}\right\rangle$ term.

Obviously, if the $\left\langle T_{z}\right\rangle$ term can be neglected then the quantities $N_{\mathrm{h}}$ and $I_{\mathrm{t}}$ drop out for the ratio $m_{l} / m_{s}$ and thus the uncertainties involved in fixing $N_{\mathrm{h}}$ (Sec. III C) or calculating $I_{\mathrm{t}}$ (Sec. III A) are no longer relevant. Therefore it has been suggested ${ }^{29}$ that a very good estimate for the real ratio $m_{l} / m_{s}$ may be obtained from $2 I_{\mathrm{m}} / 3 I_{\mathrm{s}}$. This suggestion is based on the assumptions that the $\left\langle T_{z}\right\rangle$ term can be neglected and that the further approximations underlying the derivation of the sum rules are well fulfilled. As demonstrated in Secs. III B and III A, both of these assumptions are not well fulfilled at the interface of the Co-Pt multilayer, and therefore we cannot expect to get good estimates for the real ratio $m_{l} / m_{s}$ from $2 I_{\mathrm{m}} / 3 I_{\mathrm{s}}$. To demonstrate this, we compare in Fig. 6 the directly calculated values $m_{l} / m_{s}$ with those obtained from Eq. (7). To test the general validity of Eq. (7), we first include the $\left\langle T_{z}\right\rangle$ term and calculate all quantities by taking into account only the $5 d$ contributions (small filled circles and small filled squares). It becomes obvious that the directly calculated ratios differ from those obtained by Eq. (7), which demonstrates again that the sum rules are not well fulfilled at the interface (cf. Sec. III A). The same holds when we take into account all $l$ contributions and terminate the integrals at $E_{\mathrm{c}}=E_{\mathrm{d}}$ to eliminate the $s$ contributions to the absorption signal above $E_{\mathrm{d}}$ (big open circles and big open squares). When excluding the $\left\langle T_{z}\right\rangle$ term (triangles) even the qualitative behavior of the ratio as function of the layer number $l$ is described incorrectly. Altogether, we must conclude that even the ratios $m_{l} / m_{s}$ cannot be determined reliably from the XMCD spectra for the Pt atoms in the Co-Pt multilayer. 


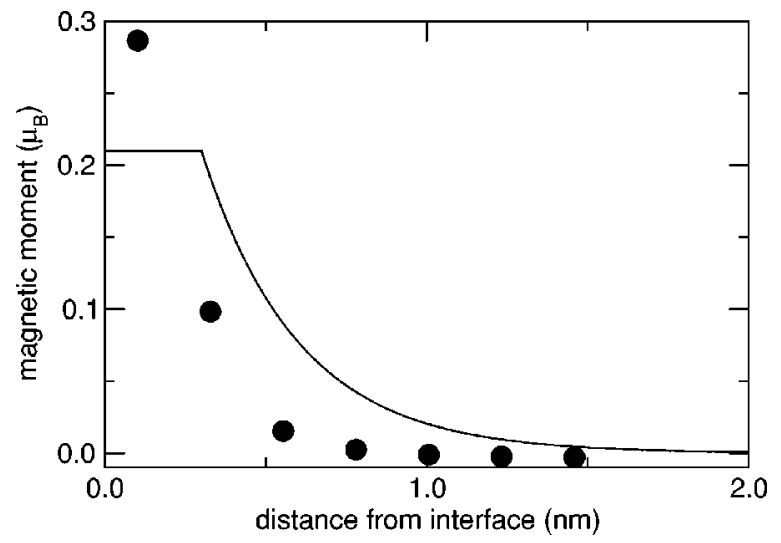

FIG. 7. Comparison of the profile of the induced Pt magnetization as obtained by the reflectometry (Ref. 2) (full line), and by the LMTO calculation (filled circles) for an ideal Co-Pt interface in $\mathrm{Co}_{2}-\mathrm{Pt}_{13}$ system.

\section{MAgNetiZATION PROFILE IN THE Pt LAYER}

As outlined in the Introduction, it is very interesting to know the profile of the induced magnetization in the Pt layer. The general profile has been investigated ${ }^{2}$ by means of the $\mathrm{x}$-ray resonant reflectometry combined with a modeling based on a magnetically modified Parratt formalism (see Sec. I). The experimental profile is given by the full line in Fig. 7. A very good agreement of the predictions of the model with the experimental asymmetry ratio of the reflectometry was obtained by a Pt depth profile starting with a constant magnetization of $(0.21 \pm 0.04) \mu_{\mathrm{B}}$ for a range of $(0.3 \pm 0.1) \mathrm{nm}$ at the Co interface, with parallel alignment between the Co and Pt moments, followed by an exponential decay. In contrast, the theoretical magnetization profile (including spin and orbital contributions) obtained for an ideal, i.e., atomically flat interface does not exhibit such an initial plateau, but it decreases continously and rapidly, starting from a value that is considerably larger than the experimental plateau value (Fig. 7). The differences between the theoretical and the experimental profiles probably arise from the fact that the real interface was not ideal because of interdiffusion, roughness, etc. Calculations for nonideal interfaces are required to test for this possible explanantion.

There is an open question in the literature ${ }^{4,5}$ concerning the sign of the induced magnetic moment for the innermost three $\mathrm{Pt}$ monolayers in a $\mathrm{Co}_{2}-\mathrm{Pt}_{13}$ multilayer. Former LMTO calculations neglecting the spin-orbit coupling ${ }^{4,5}$ predicted a significant decrease of the total moment of the Pt layer for more than 7 monolayers Pt due to magnetic moments in these innermost layers which appeared to be oriented antiparallel to the other Pt moments and to the Co moments. This decrease could not be confirmed experimentally. ${ }^{4,5}$ To investigate this in more detail, ${ }^{4,5}$ the three innermost Pt layers were replaced by Ir layers. As observed in the case of Pt and Ir impurities in $\mathrm{Fe}$, both elements show a similar XMCD effect. $^{22,23}$ From the XMCD signal it could be concluded that these Ir layers indeed couple ferromagnetically to the Co layers, and the magnetic moment per atom was estimated to be of the order of $0.01 \mu_{\mathrm{B}}$.

We now calculate directly (i.e., not via the sum rules) the

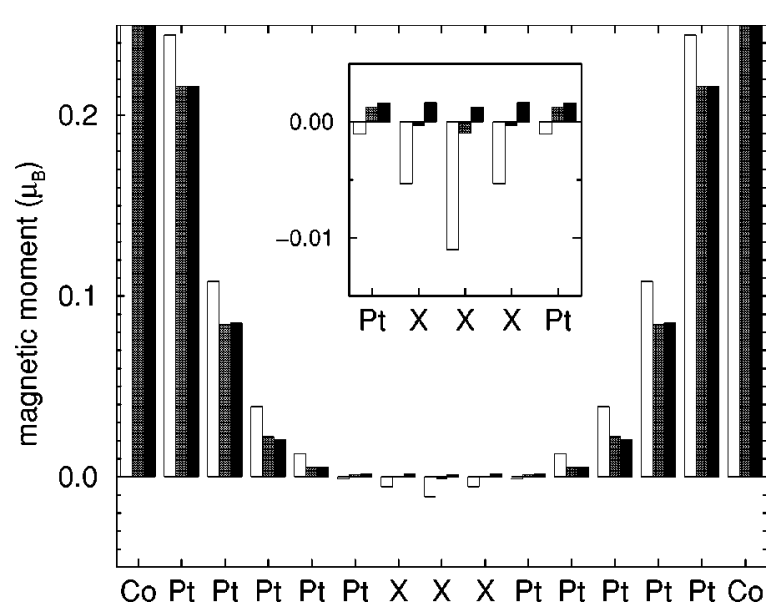

FIG. 8. Magnetic moments in a $\mathrm{Co}_{2}-\mathrm{Pt}_{13}$ multilayer as obtained by the LMTO method. White bars: $X=\mathrm{Pt}$, no spin-orbit coupling. Gray bars: $X=\mathrm{Pt}$, with spin-orbit coupling. Black bars: $X=\mathrm{Ir}$, with spin-orbit coupling. The inset represents the data for the innermost layers on a large scale.

induced magnetization profile for the $\mathrm{Co}_{2}-\mathrm{Pt}_{13}$ multilayer, and for this system with the three innermost Pt layers replaced by Ir layers (Fig. 8). The calculations are performed both with and without the spin-orbit coupling (It thereby turned out that a very accurate Brillouin-zone sampling is required to obtain converged results.) There are three main results, which are as follows.

(1) The magnetic moments are considerably influenced by spin-orbit coupling. Taking into account the spin-orbit coupling reduces the size of the innermost moments by a factor of about 10-20. Therefore, our calculation does not predict a significant decrease of the total Pt moment with increasing $\mathrm{Pt}$ layer thickness as suggested by the former calculations, ${ }^{4,5}$ in agreement with the experiments. ${ }^{4,5}$ The calculated moments for the Ir moments are about an order of magnitude smaller than the experimentally estimated moments. Accordingly, our calculations yield nearly no XMCD signal for the three Ir layers, whereas experimentally a clear signal could be observed.

(2) The remaining very small Pt moments for the three innermost layers are antiferromagnetically aligned to the Co moments.

(3) The magnetic moments of the Ir layers are ferromagnetically aligned to the Co moments, in agreement with the experiment. However, their size is much smaller than estimated from the experiments.

At the moment the reason for the discrepancy between the experimental and theoretical moment sizes is not clear. It is planned to repeat the experiments with better resolution and improved sample preparation.

Finally, we want to assess the assumption ${ }^{6,7}$ that the moments of the various Pt multilayers depend just on the distance from the interface but not on the Pt layer thickness. As outlined in the Introduction, this assumption is the basis for a layer-resolved analysis of the induced magnetization profile from the XMCD spectra obtained for various thicknesses of the Pt layer. ${ }^{6,7}$ The results of our calculations (Fig. 9) clearly demonstrate that this assumption is not well justified for 


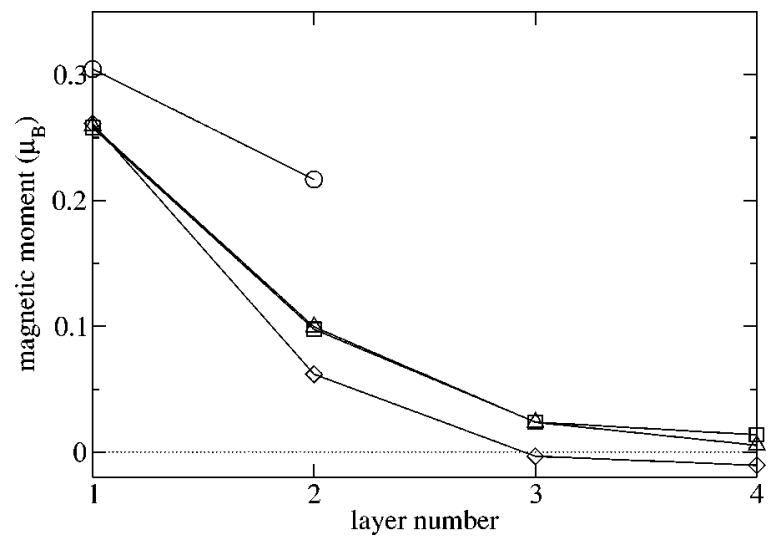

FIG. 9. The profile of the induced Pt moments as calculated by the LMTO method for $\mathrm{Co}_{2}-\mathrm{Pt}_{3}$ system (circles), $\mathrm{Co}_{2}-\mathrm{Pt}_{7}$ system (squares), $\mathrm{Co}_{2}-\mathrm{Pt}_{9}$ system (diamonds), and $\mathrm{Co}_{2}-\mathrm{Pt}_{13}$ system (triangles).

small layer thicknesses which are essential for the determination of the magnetization profile.

\section{CONCLUSIONS}

In the present paper the induced magnetic orbital and spin moments as well as the XMCD spectra are calculated for the multilayer $\mathrm{Co}_{2}-\mathrm{Pt}_{n}$ systems with $n=3,7,9,13$. The calculations are performed by the $a b$ initio density-functional electron theory and with two band-structure methods, LMTOASA as well as FLAPW. It turns out that there is a good agreement between these two types of calculations. The profile of the induced magnetic moments is obtained and compared to experimental results. It is shown that for the three innermost $\mathrm{Pt}$ layers in the $\mathrm{Co}_{2}-\mathrm{Pt}_{13}$ multilayer the magnetic moments are aligned antiferromagnetically to the Co moments, but they are very small in size. Replacing these Pt layers by Ir layers, we find a ferromagnetic orientation of the Ir moments, which is in agreement with experimental results, but the magnitudes of the moments are much smaller than those estimated from the experiments. It turns out that the $\mathrm{Pt}$ moments do not depend just on the distance from the inter- face but also on the thickness of the whole Pt layer, which throws some doubts on the method to obtain layer-resolved profiles for the induced magnetization by XMCD measurements of the multilayer systems with various thicknesses.

The main objective of the present paper is to assess for the case of induced moments close to interfaces some of the assumptions which are commonly adopted to determine the magnetic moments from the XMCD spectra via the sum rules. It turns out that for the Co-Pt system the orbital sum rule is violated by up to $35 \%$ at the interface. The spin sum rule gives much better results as long as the $\left\langle T_{z}\right\rangle$ term is included but it fails similarly badly as the orbital sum rule when the $\left\langle T_{z}\right\rangle$ term is neglected (what is done in most experiments). We therefore must conclude that the analysis of the XMCD spectra via the sum rules for the induced magnetic moments of Pt atoms close to an interface can give at best semiquantitative results. We want to note that this negative assessment for the Co-Pt layer should not be generalized to all multilayer systems with induced magnetic moments. Our guess is that a big part of the problems for Pt arise from the fact that there are only a few holes in the $5 d$ band, like in $\mathrm{Pd}(4 d)$ or $\mathrm{Ni}(3 d)$. It may well be that the application of the sum rules to systems with more $d$ holes is less critical.

Finally, we want to note there is a somewhat different experience with the application of the sum rules for surfaces of the $3 d$ metals $\mathrm{Fe}, \mathrm{Co}$, and $\mathrm{Ni}^{11,20,21}$ There it has been found that the orbital sum rule is valid to within $5-10 \%$ as long as an appropriately chosen cutoff energy is used for the integrations appearing in the sum rules. However, for the spin sum rule, large errors have been found for $\mathrm{Ni}$, even when the $\left\langle T_{z}\right\rangle$ term is taken into account. This clearly demonstrates that one should be cautious when trying to generalize statements concerning the applicability of sum rules from the experience made for selected systems.

\section{ACKNOWLEDGMENTS}

The authors are indebted to J. Kuneš for kindly supplying them with the XMCD code for WIEN97. They also acknowledge helpful discussions with J. Kuneš, P. M. Oppeneer, and H. Ebert.
*Electronic address: faehn@physix.mpi-stuttgart.mpg.de

${ }^{1}$ Y. P. Lee, R. Gontarz, and Y. V. Kudryavtsev, Phys. Rev. B 63, 144402 (2001).

${ }^{2}$ J. Geissler, E. Goering, M. Justen, F. Weigand, G. Schütz, J. Langer, D. Schmitz H. Maletta, and R. Mattheis, Phys. Rev. B 65, 020405 (2002).

${ }^{3}$ G. Schütz, W. Wagner, W. Wilhelm, P. Kienle, R. Zeller, R. Frahm, and G. Materlik, Phys. Rev. Lett. 58, 737 (1987).

${ }^{4}$ S. Rüegg, G. Schütz, P. Fischer, R. Wienke, W. B. Zeper, and H. Ebert, J. Appl. Phys. 69, 5655 (1991).

${ }^{5}$ G. Schütz, S. Stähler, M. Knülle, P. Fischer, S. Parkin, and H. Ebert, J. Appl. Phys. 73, 6430 (1993).

${ }^{6}$ P. Poulopoulos, A. Scherz, F. Wilhelm, H. Wende, and K. Baberschke, Phys. Status Solidi A 189, 293 (2002).

${ }^{7}$ J. Vogel, A. Fontaine, V. Cros, F. Petroff, J.-P. Kappler, G. Krill, A. Rogalev, and J. Goulon, Phys. Rev. B 55, 3663 (1997).
${ }^{8}$ B. T. Thole, P. Carra, F. Sette, and G. van der Laan, Phys. Rev. Lett. 68, 1943 (1992).

${ }^{9}$ P. Carra, B. T. Thole, M. Altarelli, and X. Wang, Phys. Rev. Lett. 70, 694 (1993).

${ }^{10}$ H. Ebert, Rep. Prog. Phys. 59, 1665 (1996).

${ }^{11}$ R. Wu and A. J. Freeman, Phys. Rev. Lett. 73, 1994 (1994).

${ }^{12}$ J. P. Perdew and Y. Wang, Phys. Rev. B 45, 13244 (1992).

${ }^{13}$ O. K. Andersen and O. Jepsen, Phys. Rev. Lett. 53, 2571 (1984).

${ }^{14}$ M. Liebs, K. Hummler, and M. Fähnle, Phys. Rev. B 51, 8664 (1995).

${ }^{15}$ O. Grotheer, C. Ederer, and M. Fähnle, Phys. Rev. B 63, 100401 (2001).

${ }^{16}$ P. Blaha, K. Schwarz, P. Sorantin, and S. B. Trickey, Comput. Phys. Commun. 59, 399 (1990).

${ }^{17}$ E. Wimmer, H. Krakauer, M. Weinert, and A. J. Freeman, Phys. Rev. B 24, 864 (1981). 
${ }^{18}$ J. Kuněs, P. M. Oppeneer, H.-C. Mertins, F. Schäfers, A. Gaupp, W. Gudat, and P. Novák, Phys. Rev. B 64, 174417 (2001).

${ }^{19}$ J. Kuněs, P. Novák, M. Diviš, and P. M. Oppeneer, Phys. Rev. B 63, 205111 (2001).

${ }^{20}$ R. Wu, D. Wang, and A. J. Freeman, J. Magn. Magn. Mater. 132, 103 (1994).

${ }^{21}$ R. Wu and A. J. Freeman, J. Magn. Magn. Mater. 200, 498 (1999).

${ }^{22}$ P. Wienke, G. Schütz, and H. Ebert, J. Appl. Phys. 69, 6147 (1991).

${ }^{23}$ G. Schütz, M. Knülle, and H. Ebert, Phys. Scr. T49, 302 (1993).
${ }^{24}$ A. Ankudinov and J. J. Rehr, Phys. Rev. B 51, 1282 (1995).

${ }^{25}$ W. Grange, et al., Phys. Rev. B 58, 6298 (1998).

${ }^{26}$ S. Schweizer, C. Elsässer, K. Hummler, and M. Fähnle, Phys. Rev. B 46, 14270 (1992).

${ }^{27}$ J. Stöhr and H. König, Phys. Rev. Lett. 75, 3748 (1995).

${ }^{28}$ E. Goering, A. Bayer, S. Gold, G. Schütz, M. Rabe, U. Rüdiger, and G. Güntherodt, Phys. Rev. Lett. 88, 207203 (2002).

${ }^{29}$ C. T. Chen, Y. U. Idzerda, H.-J. Lin, N. V. Smith, G. Meigs, E. Chaban, G. H. Ho, E. Pellegrin, and F. Sette, Phys. Rev. Lett. 75, 152 (1995). 